\title{
Efeitos dos programas de luz sobre desempenho, rendimento de carcaça e resposta imunológica em frangos de corte
}

\author{
[Effect of lighting programs on performance, carcass yield, and immunological \\ response of broiler chickens] \\ D.T. Moraes ${ }^{1}$, L.J.C. Lara ${ }^{1}$, N.C. Baião ${ }^{1 *}$, S.V. Cançado, M.L. Gonzalez ${ }^{2}$, \\ C.A.L. Aguilar ${ }^{1}$, A.M.Q. Lana ${ }^{1}$ \\ ${ }^{1}$ Escola de Veterinária - UFMG \\ Caixa Postal 567 \\ 30123-970 - Belo Horizonte, MG \\ ${ }^{2}$ Escola de Engenharia da UFMG - Belo Horizonte, MG
}

\begin{abstract}
RESUMO
Avaliaram-se os efeitos dos programas de luz 23L:1E (23L); crescente (CRES); 16L:8E (16L) e luz natural (NAT) sobre o desempenho, rendimento de carcaça e resposta imunológica de frangos de corte machos da linhagem Cobb-500. O período de criação foi de um a 45 dias de idade, e os tratamentos foram definidos de acordo com os programas de luz. O delineamento experimental foi o inteiramente ao acaso com quatro tratamentos e seis repetições de 35 aves cada. As aves submetidas ao programa de luz CRES apresentaram maior peso vivo em relação aos demais tratamentos $(\mathrm{P}<0,05)$. $\mathrm{O}$ consumo de ração também foi maior para as aves do tratamento CRES quando comparado aos tratamentos $23 \mathrm{~L}$ e NAT $(\mathrm{P}<0,05)$, e semelhante ao tratamento $16 \mathrm{~L}(\mathrm{P}>0,05)$. A viabilidade das aves do tratamento NAT foi maior em relação às aves do tratamento $23 \mathrm{~L}$ $(\mathrm{P}<0,05)$ e semelhante aos demais tratamentos $(\mathrm{P}>0,05)$. A conversão alimentar e o rendimento de carcaça não foram influenciados pelos tratamentos $(\mathrm{P}>0,05)$. As aves do tratamento $23 \mathrm{~L}$ apresentaram maior rendimento de peito quando comparado aos demais tratamentos $(\mathrm{P}<0,05)$. A produção de anticorpos para a doença de Newcastle não foi afetada pelos tratamentos $(\mathrm{P}>0,05)$.
\end{abstract}

Palavras-chave: frango de corte, programa de luz, desempenho, rendimento de carcaça, resposta imunológica

\begin{abstract}
The effects of lighting programs 23L:1E (23L); increasing (INC); 16L: 8E (16L) and natural light (NAT) on performance, carcass yield and immune response of Cobb-500 male broiler were evaluated. Chicks were raised from one to 45-day-old and the treatments were defined according to the lighting programs. The experiment was designed in four treatments with six repetitions of 35 birds each. Broilers of the INC lighting program showed heavier weights $(P<0.05)$. Feed consumption was higher in broilers of the INC treatment when compared to the $23 \mathrm{~L}$ and NAT $(P<0.05)$, and similar to the $16 L(P>0.05)$. The viability of the NAT treatment broilers was higher than the broilers of the $23 \mathrm{~L}(P<0.05)$ and similar to the other treatments. Feed conversion and carcass yield were not influence by the treatments $(P>0.05)$. The $23 L$ treatment broilers showed higher breast yield when compared to the other treatments $(P<0.05)$. Differences were not observed among the treatments regarding antibodies levels against Newcastle disease $(P>0.05)$.
\end{abstract}

Keywords: broiler chickens, lighting programs, performance, slaughter yield, antibodies levels

Recebido em 30 de maio de 2007

Aceito em 31 de janeiro de 2008

*Autor para correspondência (corresponding author)

E-mail: baião@vet.ufmg.br

Apoio: FAPEMIG 


\section{INTRODUÇÃO}

Os conceitos de programas de luz para frangos de corte mudaram muito com o passar do tempo. Durante muitos anos, a indústria avícola utilizou programas de luz com fotoperíodo de 23 a 24 horas de luz diária, com o objetivo de maximizar o consumo de ração e ganho de peso dos frangos de corte. Com a evolução da avicultura, o melhoramento genético proporcionou ao mercado uma ave diferente. Desta forma, surgiram muitos estudos relacionando os efeitos do fotoperíodo com os problemas de pernas, mortalidade e bem-estar das aves (Gordon, 1994).

Com o avanço nos estudos sobre programas de luz, os pesquisadores concluíram que o melhor desempenho e bem-estar das aves poderiam ser alcançados com fotoperíodos moderados, que possibilitariam aumento nas horas de sono, menor estresse fisiológico, melhora na resposta imunológica e, possivelmente, melhora no metabolismo ósseo e na condição das patas (Rutz e Bermudez, 2004; Lopez et al., 2007).

Vários estudos têm mostrado que os programas de luz podem influenciar o desempenho e o rendimento de abate em frangos de corte. Com o objetivo de se avaliar o efeito do aumento do fotoperíodo sobre o desempenho de frangos de corte, Classen e Riddell (1989) trabalharam com três programas de luz (quase contínua (23L) com 23L:1E de 0 a 42 dias; crescente (CRES) sendo $6 \mathrm{~L}: 18 \mathrm{E}$ de zero a sete dias, 10L:14E de oito a 14 dias, 14L:10E de 15 a 21 dias, $18 \mathrm{~L}: 6 \mathrm{E}$ de 22 a 28 dias e 23L:1E de 29 a 42 dias; e seis horas de luz (6L) até os 21 dias e 23 horas de luz dos 21 aos 42 dias de idade). Os autores verificaram que não houve efeito dos tratamentos sobre o ganho de peso das aves aos 42 dias de idade. O consumo de ração foi superior nas aves do tratamento $23 \mathrm{~L}$ quando comparado com o consumo das aves do tratamento CRES, e a conversão alimentar foi superior no tratamento CRES quando comparado com o tratamento $23 \mathrm{~L}$ no período de zero a 42 dias de idade das aves.

Classen et al. (1991), ao trabalharem com três programas de luz: luz quase contínua (23L:1E), crescente (aumentando-se o fotoperíodo de seis para 23 horas de luz/dia no período de quatro a 35 dias de idade das aves e fotoperíodo crescente + intermitente - com o acréscimo de uma hora de luz no meio do período de escotofase), não observaram diferenças entre os tratamentos para ganho de peso, consumo de ração e conversão alimentar aos 42 dias de idade dos frangos.

Renden et al. (1994) avaliaram o rendimento de carcaça e cortes aos 42 dias de idade dos frangos em dois programas de luz (23L:1E e 14L:10E). Em relação ao rendimento de asas e coxas, o tratamento com 14 horas de luz apresentou rendimento superior ao tratamento 23L:1E. Em relação ao rendimento de peito, o programa de luz 23L:1E apresentou melhor resultado quando comparado ao tratamento $14 \mathrm{~L}: 10 \mathrm{E}$.

Renden et al. (1996), trabalhando com programas de luz quase contínua (23L:1E), constante (16L:8E), intermitente 1 (16L:3E:1L:4E) e intermitente 2 (16L:2E:1L:2E:1L:2E), não encontraram diferenças de peso das carcaças e rendimentos de asas, sobre-coxas e peito entre os tratamentos. O rendimento de coxa das aves submetidas ao tratamento $16 \mathrm{~L}: 8 \mathrm{E}$ foi maior que o das aves do tratamento 23L:1E.

O objetivo deste trabalho foi avaliar os efeitos dos diferentes programas de luz sobre o desempenho, rendimentos de carcaça e cortes e resposta imunológica contra a doença de Newcastle em frangos de corte.

\section{MATERIAL E MÉTODOS}

O experimento foi realizado no período de 26 de agosto a 11 de outubro de 2005. As aves, alojadas em galpão experimental convencional, foram submetidas a manejo semelhante aos usados nas criações comerciais. Foram utilizados 840 pintos de corte, machos, da linhagem Cobb-500, de um dia de idade, alojando-se 35 aves por boxe $(11,66$ aves $/ \mathrm{m}^{2}$ ) com água e ração oferecidas à vontade. $\mathrm{O}$ período de criação foi de um a 45 dias de idade. As aves foram vacinadas contra as doenças de Gumboro e Newcastle aos 12 dias de idade.

Foram utilizadas lâmpadas fluorescentes compactas de 11 watts (220 volts) de potência, de cor branca, para fornecer um nível de iluminamento de 20 lux na altura das aves. Essas lâmpadas foram instaladas a uma altura de dois metros do piso de cada boxe. A verificação do nível de iluminamento foi feita utilizando-se um luxímetro eletrônico ${ }^{1}$ com escalas 20-200-2000-20000 lux, RS232, datalogger. O cálculo da iluminação foi feito de acordo com Niskier e Macintyre (2000).

O aviário foi dividido em quatro partes de acordo com os tratamentos, sendo que a divisória foi feita 
utilizando-se lonas pretas de 200 microns de espessura, para se garantir que a luz fornecida para cada tratamento não interferisse no iluminamento dos demais tratamentos.

Foram utilizados três tipos de rações de acordo com as fases de criação; inicial (um a 21 dias de idade), crescimento (22 a 40 dias de idade) e acabamento (41 a 45 dias de idade). Para a formulação das rações e cálculo dos níveis nutricionais, foram considerados os valores nutricionais dos ingredientes estabelecidos nas tabelas brasileiras de exigências nutricionais de aves e suínos (Rostagno et al., 2000). As rações, de acordo com cada fase de criação, foram as mesmas. As composições das rações com seus respectivos valores nutricionais calculados encontram-se na Tab. 1. Para o cálculo das rações, foi utilizado o programa Supercrac versão 3.1 .

Tabela 1. Composição percentual das rações e respectivos níveis nutricionais calculados

\begin{tabular}{|c|c|c|c|}
\hline Ingrediente & Inicial & Crescimento & Acabamento \\
\hline Milho moído & 54,000 & 60,000 & 65,000 \\
\hline Farelo de soja & 38,000 & 32,000 & 27,000 \\
\hline Óleo de soja & 2,400 & 3,000 & 3,600 \\
\hline Farinha de carne e ossos & 4,200 & 3,650 & 3,100 \\
\hline Sal & 0,400 & 0,400 & 0,400 \\
\hline Metionina, 99\% & 0,230 & 0,170 & 0,110 \\
\hline Lisina $-\mathrm{HCl}-99 \%$ & 0,075 & 0,075 & 0,053 \\
\hline Calcário & 0,372 & 0,430 & 0,479 \\
\hline Colina $60 \%$ & 0,070 & 0,075 & 0,058 \\
\hline Sup. vitamínico mineral ${ }^{1}$ & 0,253 & 0,200 & 0,200 \\
\hline Total & 100,000 & 100,000 & 100,000 \\
\hline \multicolumn{4}{|l|}{ Nível nutricional $^{2}$} \\
\hline Energia (kcal) & 3000 & 3110 & 3200 \\
\hline Proteína bruta (\%) & 22,10 & 20,00 & 18,00 \\
\hline Cálcio (\%) & 1,00 & 0,87 & 0,80 \\
\hline Fósforo total (\%) & 0,685 & 0,62 & 0,56 \\
\hline Fósforo disponível (\%) & 0,45 & 0,40 & 0,35 \\
\hline Lisina total $(\%)$ & 1,30 & 1,15 & 1,00 \\
\hline Met + Cis $(\%)$ & 0,94 & 0,82 & 0,71 \\
\hline Metionina (\%) & 0,58 & 0,49 & 0,41 \\
\hline Sódio (\%) & 0,22 & 0,22 & 0,21 \\
\hline
\end{tabular}

${ }^{1}$ Suplemento vitamínico - inicial - conteúdo em 1kg: Ac. fólico 800mg, Ac. pantotênico 12.500mg, Antioxidante 0,5g, Biotina 40mg, Niacina 33.600mg, Selênio 300mg, Vit. A $6.700 .000 U I$, Vit. B1 $1.750 \mathrm{mg}$, Vit. B12 9.600mcg, Vit. B2 4.800mg, Vit. B6 2.500mg, Vit. D3 1.600.000UI, Vit. E 14.000mg, Vit. K3 1.440mg. Suplemento mineral conteúdo em $0,5 \mathrm{~kg}$ : Manganês $150.000 \mathrm{mg}$, Zinco $100.000 \mathrm{mg}$, Ferro $100.000 \mathrm{mg}$, Cobre $16.000 \mathrm{mg}$, Iodo $1.500 \mathrm{mg}$. Promotor de crescimento e coccidicida: Surmax 100 (Avilamicina) 80ppm, Halquinol 50ppm, Monteban G 100 (Narasina) 600ppm, Nicarbazina 25\% 200ppm.

${ }^{1}$ Suplemento vitamínico - crescimento - conteúdo em 1kg: Ac. fólico 650mg, Ac. pantotênico 10.400mg, Antioxidante 0,5g, Niacina 28.000mg, Selênio 300mg, Vit. A 5.600.000UI, Vit. B1 0,550mg, Vit. B12 8.000mcg, Vit. B2 4.000mg; Vit. B6 2,080mg, Vit. D3 1.200.000UI, Vit. E 10.000mg, Vit. K3 1.200mg. Suplemento mineral conteúdo em 0,5kg: Manganês $150.000 \mathrm{mg}$, Zinco $100.000 \mathrm{mg}$, Ferro $100.000 \mathrm{mg}$, Cobre $16.000 \mathrm{mg}$, Iodo $1.500 \mathrm{mg}$. Promotor de crescimento e coccidicida: Surmax 100 (Avilamicina) 80ppm, Halquinol 50ppm, Coccibac SDT (Sulfaquinoxalina + Diaveridina + Trimetropim) 100ppm; Coxistac 12\% (Salinomicina) 550ppm.

${ }^{1}$ Suplemento vitamínico - acabamento - conteúdo em $1 \mathrm{~kg}$ : Ac. pantotênico 7.070mg, Antioxidante 0,5g, Niacina $20.400 \mathrm{mg}$, Selênio 200mg, Vit. A $1.960 .000 \mathrm{UI}$, Vit. B12 4.700mcg, Vit. B2 2.400mg, Vit. D3 550.000UI, Vit. E $5.500 \mathrm{mg}$, Vit. K3 550mg. Suplemento mineral - conteúdo em 0,5kg: Manganês $150.000 \mathrm{mg}$, Zinco 100.000mg, Ferro $100.000 \mathrm{mg}$, Cobre $16.000 \mathrm{mg}$, Iodo $1.500 \mathrm{mg}$. Promotor de crescimento e coccidicida: Surmax 100 (Avilamicina) 80ppm, Coxistac 12\% (Salinomicina) 500ppm.

Rostagno et al. (2000)

${ }^{1}$ LDR-380 - Instrutherm - São Paulo, Brasil. 
Os tratamentos foram definidos de acordo com os programas de luz, os quais foram caracterizados segundo a Tab. 2 .

Todas as aves foram pesadas aos sete e aos 45 dias de idade. As variáveis estudadas foram: peso vivo (PV), consumo de ração (CR), conversão alimentar (CA), taxa de viabilidade (VIAB), rendimento de carcaça (RC) e seus cortes (peito, coxas e asas).

Aos 45 dias de idade, após a obtenção do peso médio dos frangos, uma amostra de cinco aves por repetição foi escolhida aleatoriamente para avaliação dos rendimentos de abate. Após a identificação individual, as 30 aves selecionadas permaneceram em jejum pelo período ideal de 10 horas (Schettino et al., 2006) e, logo após esse período, foram abatidas. $\mathrm{O}$ rendimento de carcaça foi considerado em relação ao peso vivo em jejum obtido antes do abate, na plataforma do abatedouro. Para as avaliações dos cortes (peito, coxas + sobrecoxas e asas), o rendimento foi considerado em relação ao peso da carcaça eviscerada.

Tabela 2. Período de iluminamento e de escuro de acordo com os tratamentos

\begin{tabular}{ccccc}
\hline Idade & \multicolumn{4}{c}{ Tratamentos } \\
\cline { 2 - 5 } (dias) & $23 \mathrm{~L}$ & CRES & $16 \mathrm{~L}$ & NAT \\
\hline 0 a 7 & $23 \mathrm{~L}: 1 \mathrm{E}$ & $23 \mathrm{~L}: 1 \mathrm{E}$ & $16 \mathrm{~L}: 8 \mathrm{E}$ & Luz natural \\
8 a 14 & $23 \mathrm{~L}: 1 \mathrm{E}$ & Luz natural & $16 \mathrm{~L}: 8 \mathrm{E}$ & Luz natural \\
15 a 21 & $23 \mathrm{~L}: 1 \mathrm{E}$ & Luz natural & $16 \mathrm{~L}: 8 \mathrm{E}$ & Luz natural \\
22 a 28 & $23 \mathrm{~L}: 1 \mathrm{E}$ & $14 \mathrm{~L}: 10 \mathrm{E}$ & $16 \mathrm{~L}: 8 \mathrm{E}$ & Luz natural \\
29 a 35 & $23 \mathrm{~L}: 1 \mathrm{E}$ & $18 \mathrm{~L}: 6 \mathrm{E}$ & $16 \mathrm{~L}: 8 \mathrm{E}$ & Luz natural \\
36 a 42 & $23 \mathrm{~L}: 1 \mathrm{E}$ & $23 \mathrm{~L}: 1 \mathrm{E}$ & $16 \mathrm{~L}: 8 \mathrm{E}$ & Luz natural \\
42 a 45 & $23 \mathrm{~L}: 1 \mathrm{E}$ & $23 \mathrm{~L}: 1 \mathrm{E}$ & $16 \mathrm{~L}: 8 \mathrm{E}$ & Luz natural \\
\hline
\end{tabular}

$\mathrm{L}=$ luz, $\mathrm{E}=$ escuro, $\mathrm{CRES}=$ crescente, $\mathrm{NAT}=$ luz natural - sem fornecimento de iluminamento artificial.

Também aos 45 dias de idade, foram coletadas amostras de sangue de duas aves por boxe, para a detecção de anticorpos para a doença de Newcastle, por meio do teste de inibição da hemaglutinação.

O delineamento experimental foi o inteiramente ao acaso, constituído por quatro tratamentos com seis repetições cada um. Para as análises dos dados de desempenho, cada repetição foi composta por 35 aves. Para as avaliações dos rendimentos de carcaça e seus cortes, o delineamento experimental foi o mesmo utilizado para o desempenho, e cada ave foi considerada como uma repetição, totalizando 120 aves, sendo 30 repetições por tratamento. Para a análise de produção de anticorpos, cada ave foi considerada uma repetição, obtendo-se uma amostra de 12 aves por tratamento. Os dados foram submetidos à análise de variância, e as médias comparadas pelo teste Student Newman Keuls, tanto para a avaliação de desempenho quanto para as análises dos rendimentos de carcaças, cortes e títulos de anticorpos (Sampaio,
2002). As análises estatísticas foram realizadas utilizando-se o SAS software (User's..., 1986).

\section{RESULTADOS E DISCUSSÃO}

Os resultados referentes ao peso vivo (PV), consumo de ração (CR), conversão alimentar (CA) e taxa de viabilidade (VIAB) dos pintos de um a sete dias de idade se encontram na Tab. 3. Os pintos submetidos ao programa de luz 23L e CRES apresentaram maior PV em relação aos demais tratamentos $(\mathrm{P}<0,05)$. Este resultado deve-se ao fato de os pintos desses dois tratamentos estarem expostos a maior fotoperíodo (23L:1E), o que possibilitou maior tempo de acesso aos comedouros. Estes resultados foram semelhantes aos encontrados por Renden et al. (1992) e Renden et al. (1993), que observaram PV maior para aves submetidas ao tratamento $23 \mathrm{~L}: 1 \mathrm{E}$ em relação ao tratamento 16L:8E. Em outro experimento, Renden et al. (1994) encontraram resultados diferentes, em que as aves submetidas a 23 horas de luz apresentaram PV semelhante às aves com 16 horas de luz. 
Efeitos dos programas de luz...

Tabela 3. Desempenho dos pintos no período de um a sete dias de idade de acordo com os diferentes programas de luz

\begin{tabular}{ccccc}
\hline Tratamento & PV $(\mathrm{g})$ & CR $(\mathrm{g})$ & CA $(\mathrm{g} / \mathrm{g})$ & VIAB $(\%)$ \\
\hline 23L & $194,8 \mathrm{a}$ & $162,1 \mathrm{a}$ & $0,832 \mathrm{c}$ & $99,03 \mathrm{a}$ \\
CRES & $198,5 \mathrm{a}$ & $163,5 \mathrm{a}$ & $0,822 \mathrm{bc}$ & $99,51 \mathrm{a}$ \\
16L & $187,8 \mathrm{~b}$ & $151,3 \mathrm{~b}$ & $0,806 \mathrm{ab}$ & $100,00^{*}$ \\
NAT & $182,5 \mathrm{~b}$ & $144,6 \mathrm{c}$ & $0,793 \mathrm{a}$ & $100,00^{*}$ \\
\hline CV $(\%)$ & 2,83 & 3,08 & 1,97 & 0,95
\end{tabular}

Médias seguidas de letras distintas na coluna diferem entre si pelo teste $\mathrm{SNK}(\mathrm{P}<0,05)$.

$23 \mathrm{~L}=23$ horas de luz, CRES $=$ luz crescente, $16 \mathrm{~L}=16$ horas de luz e 8 de escuro; NAT $=$ luz natural $\mathrm{PV}=$ peso vivo, $\mathrm{CR}=$ consumo de ração, $\mathrm{CA}=$ conversão alimentar, $\mathrm{VIAB}=$ taxa de viabilidade. *dados não submetidos à análise estatística por não apresentarem variabilidade.

O CR das aves submetidas ao programa NAT foi menor em relação aos demais tratamentos $(\mathrm{P}<0,05)$. As aves do tratamento $16 \mathrm{~L}$ apresentaram maior $\mathrm{CR}$ quando comparado ao tratamento NAT e menor que os tratamentos $23 \mathrm{~L}$ e CRES $(\mathrm{P}<0,05)$. Tais resultados se devem ao tempo de acesso aos comedouros. Dessa forma, aves submetidas a maior fotoperíodo (tratamentos 23L e CRES) apresentaram maior $\mathrm{CR}$ quando comparadas às aves submetidas ao fotoperíodo de 16 horas (16L), as quais também tiveram maior consumo em relação às aves submetidas ao tratamento natural (aproximadamente 12 horas de luz).

A CA foi maior nos pintos submetidos ao tratamento NAT quando comparado com as aves dos tratamentos $23 \mathrm{~L}$ e CRES e menor nas aves do tratamento 23L quando comparado aos tratamentos 16L e NAT (Tab. 3 - P<0,05). Provavelmente, esse fato se deve ao nível de atividade das aves, ou seja, quanto maior o fotoperíodo, maior a movimentação das aves e maior o gasto de energia, levando à menor conversão alimentar. De acordo com Wenk e Van Es (1976), citados por Ohtani e Leeson (2000), o gasto de energia na realização de atividades físicas para aves em crescimento é em torno de 7 a $15 \%$ da energia metabolizável consumida. Em relação à VIAB, não foram observadas diferenças entre os tratamentos $23 \mathrm{~L}$ e CRES $(\mathrm{P}>0,05)$.

Os resultados referentes ao $\mathrm{PV}, \mathrm{CR}, \mathrm{CA}$ e VIAB das aves de um a 45 dias de idade encontram-se descritos na Tab. 4.

Tabela 4. Desempenho dos frangos no período de um a 45 dias de idade de acordo com os diferentes programas de luz

\begin{tabular}{ccccc}
\hline Tratamento & PV $(\mathrm{g})$ & CR $(\mathrm{g})$ & CA $(\mathrm{g} / \mathrm{g})$ & VIAB $(\%)$ \\
\hline 23L & $3393 \mathrm{~b}$ & $5622 \mathrm{~b}$ & $1,657 \mathrm{a}$ & $87,3 \mathrm{~b}$ \\
CRES & $3486 \mathrm{a}$ & $5808 \mathrm{a}$ & $1,668 \mathrm{a}$ & $88,9 \mathrm{ab}$ \\
$16 \mathrm{~L}$ & $3409 \mathrm{~b}$ & $5706 \mathrm{ab}$ & $1,673 \mathrm{a}$ & $93,2 \mathrm{ab}$ \\
NAT & $3340 \mathrm{~b}$ & $5656 \mathrm{~b}$ & $1,694 \mathrm{a}$ & $95,6 \mathrm{a}$ \\
\hline CV $(\%)$ & 1,57 & 1,52 & 1,46 & 5,22 \\
\hline
\end{tabular}

Médias seguidas de letras distintas na coluna diferem entre si pelo teste $\mathrm{SNK}(\mathrm{P}<0,05)$.

$23 \mathrm{~L}=23$ horas de luz, CRES $=$ luz crescente, $16 \mathrm{~L}=16$ horas de luz e 8 de escuro, $\mathrm{NAT}=$ luz natural $\mathrm{PV}=$ peso vivo, $\mathrm{CR}=$ consumo de ração, $\mathrm{CA}=$ conversão alimentar, $\mathrm{VIAB}=$ taxa de viabilidade.

O PV das aves foi maior no tratamento CRES quando comparado com os demais tratamentos $(\mathrm{P}<0,05)$, os quais apresentaram $\mathrm{PV}$ semelhantes entre si (Tab. 4 - P>0,05). Ao se comparar o PV de aves submetidas a programas de luz crescentes com programa de luz contínua, é esperado que as aves dos programas de luz crescentes resultem em menor crescimento inicial, porém, com tendência, ao final do período de criação, de apresentarem pesos semelhantes e até superiores aos pesos das aves submetidas aos programas contínuos de luz. Este resultado pode ser explicado pelo fato de que aves submetidas ao aumento do fotoperíodo apresentam produção de hormônios anabolizantes semelhante às aves adultas, o que provoca um crescimento compensatório (Classen, 1996). Além disso, o consumo das aves do tratamento CRES foi maior em relação ao consumo das aves dos tratamentos $23 \mathrm{~L}$ e NAT e 
a CA foi semelhante em todos os tratamentos, isto também explica o maior peso das aves do tratamento CRES. Estes resultados estão de acordo com Rozenboim et al. (1999) que encontraram maior PV em frangos submetidos a tratamento de luz crescente quando comparados com frangos submetidos a tratamento de luz 23L aos 49 dias de idade. Renden et al. (1993) encontraram resultados discordantes, em que o PV foi semelhante em aves submetidas aos tratamentos 23L, 16L, 14L e CRES aos 48 dias de idade.

O CR também foi influenciado pelos tratamentos (Tab. 4 - $\mathrm{P}<0,05$ ). As aves do tratamento CRES apresentaram consumo semelhante às do tratamento $16 \mathrm{~L}$, porém consumiram mais ração que as dos tratamentos 23L e NAT. Este maior consumo das aves do tratamento CRES poderia ser justificado pelo condicionamento ao crescimento do fotoperíodo e pelo fato de estas terem apresentado maior crescimento (PV) aos 45 dias de idade, resultado do maior CR.

Os resultados de CA não foram influenciados pelos tratamentos $(\mathrm{P}>0,05)$. Stanley et al. (1997) afirmaram que um aumento no período de alimentação das aves mediante controle do fotoperíodo não necessariamente leva à maior
CA. Resultados semelhantes foram encontrados por Renden et al. (1992), Renden et al. (1993) e Renden et al. (1996).

Em relação à VIAB, foram encontradas diferenças entre os tratamentos $(\mathrm{P}<0,05)$. A VIAB do tratamento NAT foi semelhante à dos tratamentos CRES e 16L e maior que a do tratamento 23L. Este resultado pode ser explicado pelo crescimento inicial mais lento das aves do tratamento NAT, pois, em aves com crescimento lento, a ocorrência de doenças metabólicas, tais como ascite e morte súbita, é menor. Os tratamentos CRES e 16L apresentaram taxas de viabilidade semelhantes aos tratamentos 23L e NAT $(\mathrm{P}>0,05)$. Estes dados estão de acordo com Renden et al. (1992) e Renden et al. (1996) que não encontraram diferenças de mortalidade entre os programas de luz $23 \mathrm{~L}$ e $16 \mathrm{~L}$ aos 49 dias de idade das aves. Classen e Riddell (1989) e Renden et al. (1993), entretanto, observaram maior mortalidade em aves submetidas ao tratamento de luz 23L em relação às aves submetidas ao tratamento de luz crescente.

Os resultados referentes ao rendimento de carcaça, peito, coxa + sobrecoxa e asa encontram-se na Tab. 5.

Tabela 5. Rendimento de carcaça e cortes dos frangos com 45 dias de idade submetidos a diferentes programas de luz

\begin{tabular}{ccccc}
\hline Tratamento & RC $(\%)$ & Peito $(\%)$ & Coxa $(\%)$ & Asa $(\%)$ \\
\hline 23L & $86,54 \mathrm{a}$ & $34,61 \mathrm{a}$ & $28,68 \mathrm{a}$ & $8,43 \mathrm{a}$ \\
CRES & $86,95 \mathrm{a}$ & $32,82 \mathrm{~b}$ & $28,43 \mathrm{ab}$ & $8,45 \mathrm{a}$ \\
16L & $86,48 \mathrm{a}$ & $32,04 \mathrm{~b}$ & $27,86 \mathrm{~b}$ & $8,17 \mathrm{ab}$ \\
NAT & $87,89 \mathrm{a}$ & $31,89 \mathrm{~b}$ & $27,64 \mathrm{~b}$ & $7,95 \mathrm{~b}$ \\
\hline CV $(\%)$ & 3,52 & 4,80 & 4,69 & 7,06 \\
\hline
\end{tabular}

Médias seguidas de letras distintas na coluna diferem entre si pelo teste $\mathrm{SNK}(\mathrm{P}<0,05)$.

$23 \mathrm{~L}=23$ horas de luz, CRES $=$ luz crescente, $16 \mathrm{~L}=16$ horas de luz e 8 de escuro, $\mathrm{NAT}=$ luz natural, $\mathrm{RC}=$ rendimento de carcaça, Coxa $=$ coxa + sobrecoxa.

Os resultados de rendimento de carcaça não apresentaram diferenças significativas entre os tratamentos $(\mathrm{P}>0,05)$. Estes dados estão de acordo com Renden et al. (1991) e Renden et al. (1992).

Em relação ao rendimento de peito, as aves submetidas ao programa de luz 23L apresentaram maior rendimento em relação aos demais tratamentos $(\mathrm{P}<0,05)$, os quais foram semelhantes entre si. Estes resultados são semelhantes aos observados por Renden et al.
(1992), Renden et al. (1993) e Renden et al. (1994) que verificaram maior rendimento de peito em aves submetidas ao tratamento $23 \mathrm{~L}$ em relação ao tratamento $16 \mathrm{~L}$ aos 49 dias de idade. Porém, Renden et al. (1991) e Renden et al. (1996) não observaram diferenças em rendimento de peito entre os diferentes programas de luz.

Os rendimentos de coxas + sobrecoxas foram influenciados pelos tratamentos. $\mathrm{O}$ tratamento $23 \mathrm{~L}$ apresentou maior rendimento quando 
comparado aos tratamentos 16L e NAT $(\mathrm{P}<0,05)$. Estes resultados diferem dos encontrados por Renden et al. (1991), Renden et al. (1992), Renden et al. (1993) e Renden et al. (1994) que não observaram efeito dos programas de luz sobre os rendimentos de coxas. Renden et al. (1996), entretanto, verificaram maior rendimento de coxa + sobrecoxa em aves submetidas ao tratamento $16 \mathrm{~L}$ em relação às do tratamento de 23L.

O rendimento de asa foi menor no tratamento NAT quando comparado aos tratamentos $23 \mathrm{~L}$ e
CRES $(\mathrm{P}<0,05)$, sendo que os tratamentos $23 \mathrm{~L}$, CRES e 16L apresentaram rendimentos semelhantes entre si $(\mathrm{P}>0,05)$. Estes resultados foram semelhantes aos encontrados por Renden et al. (1991) e Classen (1996), mas foram diferentes aos observados por Renden et al. (1993) e Renden et al. (1996).

Os valores dos títulos médios geométricos de anticorpos para a doença de Newcastle aos 45 dias de idade das aves encontram-se na Tab. 6.

Tabela 6. Inibição da hemaglutinação - títulos médios geométricos de anticorpos (GMT) para a doença de Newcastle em aves aos 45 dias de idade

\begin{tabular}{cccccc}
\hline Tratamentos & $23 \mathrm{~L}$ & CRES & $16 \mathrm{~L}$ & NAT & CV (\%) \\
\hline GMT & $38,05 \mathrm{a}$ & $28,51 \mathrm{a}$ & $38,05 \mathrm{a}$ & $23,97 \mathrm{a}$ & 39,62 \\
\hline
\end{tabular}

Médias seguidas de letras distintas diferem entre si pelo teste $\operatorname{SNK}(\mathrm{p}<0,05)$.

$23 \mathrm{~L}=23$ horas de luz, $\mathrm{CRES}=$ luz crescente, $16 \mathrm{~L}=16$ horas de luz e 8 de escuro, $\mathrm{NAT}=$ luz natural.

$\mathrm{Na}$ avaliação dos títulos médios geométricos de anticorpos para a doença de Newcastle, realizada aos 45 dias de idade das aves, não foi encontrada diferença entre os tratamentos $(\mathrm{P}>0,05)$. Este resultado se deve, provavelmente, ao baixo desafio viral no local do experimento. Davis et al. (1997) encontraram títulos mais altos de anticorpos em aves submetidas a um programa de luz constante (16L) quando comparadas com aves submetidas a um programa de luz quase contínuo (23L). Segundo esses autores, os resultados se devem ao fato de as aves do programa de luz 16L terem ficado expostas a oito horas de escuro por dia, o que permitiu um período de descanso e uma maior produção de melatonina, resultando em melhora no sistema imunológico e, conseqüentemente, em maior produção de anticorpos.

\section{CONCLUSÃO}

O programa de luz crescente é o mais indicado para frangos de corte.

\section{AGRADECIMENTOS}

Aos senhores Guilherme e Elísio Capanema da Silva, por terem colocado à disposição dos autores a infra-estrutura de sua empresa.

\section{REFERÊNCIAS BIBLIOGRÁFICAS}

CLASSEN, H.L. Principios sobre el manejo de luz en pollos de engorde. Avicult. Profes., v.14, p.22-27, 1996.

CLASSEN, H.L.; RIDDELL, C. Photoperiodic effects on performance and leg abnormalities in broiler chickens. Poult. Sci., v.68, p.873-879. 1989.

CLASSEN, H.L.; RIDDELL, C.; ROBINSON, F.E. Effects of increasing photoperiod length on performance and health of broiler chickens. $\mathrm{Br}$. Poult. Sci., v.32, p.21-29. 1991.

DAVIS, J.F.; BROWN, T.P.; SIOPES, T. More evidence for light-dark growing. Broiler Ind., v.60, p.31-32, 1997.

GORDON, S.H. Effects of daylength and increasing daylength programs on broiler welfare and performance. World's Poult. Sci. J., v.50, p.269-282, 1994.

LOPEZ, C.A.A.; BAIÃO, N.C.; LARA, L.J.C. et al. Efeitos da forma física da ração sobre a digestibilidade dos nutrientes e desempenho de frangos de corte. Arq. Bras. Med. Vet. Zootec., v.59, p.1006-1013, 2007.

NISKIER, J.; MACINTYRE, A.J. Instalações Elétricas. 4.ed., Rio de Janeiro: LTD, 2000, p.241-306. 
OHTANI, S.; LEESON, S. The effect of intermittent lighting on metabolizable energy intake and heat production of male broilers. Poult. Sci., v.79, p.167-171, 2000.

RENDEN, J.A.; BILGILI, S.F.; KINCAID, S.A. Live performance and carcass yield of broiler strain crosses provided either sixteen or twentythree hours of light per day. Poult. Sci., v.71, p.1427-1435, 1992.

RENDEN, J.A.; BILGILI, S.F.; KINCAID, S.A. Research note: Comparison of restricted and increasing light programs for male broiler performance and carcass yield. Poult. Sci., v.72, p.378-382, 1993.

RENDEN, J. A.; BILGILI, S. F.; LIEN, R. J. et al. Live performance and yields of broilers provided various lighting schedules. Poult. Sci., v.70, p.2055-2062, 1991.

RENDEN, J.A.; MORAN, E.T.; KINCAID, S.A. Lack of interactions between dietary lysine or strain cross and photoschedule for male broiler performance and carcass yield. Poult. Sci., v.73, p.1651-1662, 1994.

RENDEN, J.A.; MORAN JR., E.T.; KINCAID, S.A. Lighting programs for broilers that reduce leg problems without loss of performance or yield. Poult. Sci., v.75, p.1345-1350, 1996.

ROSTAGNO, H.S.; ALBINO, L.F.T.; DONZELE, J.L. et al. Tabelas brasileiras para aves e suínos. Composição de alimentos $\mathrm{e}$ exigências nutricionais. Viçosa: UFV, 2000, $141 \mathrm{p}$.
ROZEMBOIM, I.; ROBINZON, B.; ROSENSTRAUCH, A. Effect of light source and regimen no growing broilers. Br. Poult. Sci., v.40, p.452-457, 1999.

RUTZ, F.; BERMUDEZ, V.L. Fundamentos de um programa de luz para frangos de corte. In: MENDES, A.A.; NAAS, I.A.; MACARI,M. (Eds). Produção de frangos de corte, Campinas: FACTA, 2004. p.157-168.

SAMPAIO, I.B.M. Estatística aplicada à experimentação animal. 2.ed., Belo Horizonte: FEPMVZ, 2002. 244p.

SCHETTINO, D.N.; CANÇADO, S.V.; BAIÃO, N.C. et al. Efeito do período de jejum pré-abate sobre o rendimento de carcaça de frango de corte. Arq. Bras. Med. Vet. Zootec., v.58, p.918924, 2006.

STANLEY, V.G.; GUTIERREZ, J.; PARKS, A.L.; et al. Relationship between age of commercial broiler chickens and response to photostimulation. Poult. Sci., v.76, p.306-310, 1997.

USER'S guide. Version 6. Cary, NC: SAS Institute, 1996.

WENK, C.; VAN ES, A.J.H. Energy metabolism of growing chickens as related to their physical activity. In: SYMPOSIUM OF THE ENERGY METABOLISM OF FARM ANIMALS, 7., 1976, Vichy. Proceedings..., Vichy, 1976. p.189192. 